\title{
FAISABILITÉ DE L'UTILISATION DES CENDRES DE CHAUDIÈRE INDUSTRIELLE DANS LE DOSAGE DU BÉTON STRUCTUREL
}

\section{ARTICLE ORIGINAL}

JUSTINO, Lucas Diego de Souza ${ }^{1}$

JUSTINO, Lucas Diego de Souza. Faisabilité de l'utilisation des cendres de chaudière industrielle dans le dosage du béton structurel. Revista Científica Multidisciplinar Núcleo do Conhecimento. An 06, Ed. 09, Vol. 02, p. 81 à 97. Septembre 2021. ISSN : 2448-0959, Lien d'accès: https://www.nucleodoconhecimento.com.br/travaux-publics/beton-structurel, DOI: 10.32749/nucleodoconhecimento.com.br/travaux-publics/beton-structurel

\section{RÉSUMÉ}

L'industrialisation et l'accélération de la croissance démographique génèrent des effets secondaires sur divers aspects sociaux, et la question environnementale est préoccupante en raison des impacts causés par l'évolution sociale. La gestion des déchets industriels est un grand défi qui implique à la fois le contrôle de leur production et l'élimination appropriée, assurant la durabilité environnementale. Les résidus de cendres de chaudière se trouvent en abondance dans les usines qui utilisent cet équipement pour la production de vapeur. Cette abondance se produit en raison de l'absence d'un endroit pour l'élimination ou la réutilisation appropriée du résidu. Compte tenu de ce scénario, cet article avait pour guide une question : seraitil possible d'utiliser ce résidu dans la production de béton structurel ? L'objectif de cette étude était de classer le résidu en définissant sa forme possible d'utilisation dans le dosage du béton et d'effectuer des dosages expérimentaux avec l'utilisation de cendres de chaudière industrielles pour évaluer ses éventuelles contributions techniques aux propriétés de base du béton. Pour cela, des échantillons ont été

\footnotetext{
${ }^{1}$ Diplômé en génie civil. ORCID : 0000-0003-2630-8866
}

RC: 97180

Disponible en: https://www.nucleodoconhecimento.com.br/travaux-publics/betonstructurel 
prélevés par une industrie installée dans la ville d'Uberlândia, dans l'État de Minas Gerais, active dans plusieurs secteurs tels que l'agriculture, la nutrition animale, les produits pharmaceutiques et autres. La classification des cendres de chaudière a été réalisée en appliquant les procédures et paramètres normatifs brésiliens utilisés pour la classification des liants et des agrégats pour le béton. Compte tenu de sa courbe granulométrique et de sa densité, le résidu a été classé comme agrégat léger et très mince, adoptant ainsi la méthodologie du remplacement partiel de l'agrégat mince par des cendres de chaudière. Il a été vérifié qu'il y avait une réduction de la maniabilité du béton à l'état frais proportionnellement à la teneur en résidus utilisée. Par conséquent, il est nécessaire d'utiliser des additifs superplastifiants dans ces cas pour maintenir la maniabilité attendue. Une réduction de la densité du béton a été remarquée lors de l'utilisation du résidu, en considérant comme nécessaire l'attention portée à cette propriété du béton par rapport à la teneur utilisée du résidu dans le dosage. II a également été observé lors de la comparaison des dosages avec l'utilisation du résidu à la dose standard, qu'il y avait une réduction de la force de compression. Cependant, il n'y avait pas de grande variation dans la force de compression entre les dosages avec différents niveaux de substitution utilisés. II a donc été conclu, compte tenu des niveaux utilisés dans cette étude, que l'utilisation de ce résidu dans la production de béton structurel était réalisable.

Mots-clés : Béton, Biomasse, Cendres de chaudière industrielle.

\section{INTRODUCTION}

La révolution industrielle commencée en Angleterre au 18ème siècle à partir de la création de la machine à vapeur par l'ingénieur écossais James Watt, a stimulé l'industrialisation britannique afin de transformer le système de production. Cette période selon Cavalcante (2011) « a été le précurseur du capitalisme, c'est-à-dire la transition du capitalisme commercial au capitalisme industriel ». Cela signifie que, dans ce contexte, le régime de fabrication, précédemment utilisé, est remplacé par le régime de facturation maquino, ce qui se traduit par des processus de production

RC: 97180

Disponible en: https://www.nucleodoconhecimento.com.br/travaux-publics/betonstructurel 
beaucoup plus agiles capables de mettre en pratique la production de masse, assurant un plus grand approvisionnement en produits et de faibles coûts de production, par rapport au scénario précédent.

Les transformations qui ont eu lieu pendant la révolution industrielle se sont étendues à l'échelle mondiale et ont accompagné l'évolution de la société au fil du temps, modernisant et améliorant les pratiques de production au fil des ans. Cependant, avec les progrès du processus d'industrialisation, la croissance de la population mondiale et par conséquent la forte demande de produits, la consommation de ressources naturelles augmente proportionnellement à cette demande, puisque ces ressources servent de matière première pour les processus de fabrication.

Le processus de croissance accélérée génère donc, comme effets secondaires, les impacts environnementaux causés par l'exploitation des ressources naturelles, sans une gestion appropriée axée sur l'entretien et la conservation de l'environnement. " L'exploitation des ressources naturelles est devenue prédatrice au profit de l'obtention de capitaux » (GANZALA, 2018).

Les effets négatifs de l'industrialisation tout au long des $20 \mathrm{e}$ et $21 \mathrm{e}$ siècles liés à l'environnement ne sont pas seulement liés à la consommation non durable des ressources naturelles impliquées dans les processus de production, mais aussi à la pollution de l'eau, du sol et de l'air causée par les déchets ou les déchets générés par les processus industriels. Cette réalité est observée tant dans les pays développés que dans les pays en développement.

Plusieurs procédés industriels utilisent des équipements appelés chaudières de production de vapeur dans le processus de production de diverses industries du sucre et de l'alcool, les usines de séchage et d'étuvage de céréales, de réfrigérateurs, l'industrie métallurgique, le textile et dans le secteur de la production d'énergie. Le type de chaudière le plus couramment utilisé actuellement est la

RC: 97180

Disponible en: https://www.nucleodoconhecimento.com.br/travaux-publics/betonstructurel 
chaudière dite à biomasse. Ces chaudières utilisent comme matériau de combustion les différentes formes de biomasse telles que: le pin, l'eucalyptus, l'écorce de riz et la bagasse de canne à sucre. Après avoir brûlé la biomasse, les cendres de chaudière sont générées sous forme de déchets, de sorte que les cendres de chaudière sont constituées de la partie résistante aux brûlures, présente dans la composition de la biomasse utilisée comme combustible dans les chaudières industrielles.

Les industries qui utilisent les chaudières, visant l'efficacité énergétique et le facteur économique, appliquent des contrôles des processus de combustion de la biomasse, ce qui réduit autant que possible la production de ce type de déchets. Cependant, la production de déchets est inévitable dans ce processus. Par conséquent, la disponibilité de ce résidu devient abondante. Dans ces industries, d'importants gisements de ce matériau sont accumulés en raison du manque d'alternatives pour la destination ou la réutilisation de celui-ci. Ces gisements occupent de grands espaces physiques dans les usines de production, qui pourraient être mieux utilisés, compte tenu de l'activité finale de l'industrie en question.

Dans le scénario de haute disponibilité du résidu de chaudière grise et de l'absence d'un site d'élimination approprié ou d'une activité pour la réutilisation du résidu, la question fondamentale se pose pour le développement de cette étude: serait-il possible d'utiliser ce résidu dans la production de béton structurel? Compte tenu du rythme accéléré du secteur de la construction et de sa recherche d'innovations, les déchets générés pourraient être réutilisés dans ce segment comme alternative durable, avec la possibilité de promouvoir des avantages techniques et économiques à la fois pour l'industrie productrice de déchets et les entreprises productrices de béton.

La contribution environnementale est la réalisation principale et la plus significative avec l'utilisation des résidus de cendres des chaudières industrielles dans la production de béton structurel, car il y aurait une double contribution environnementale pour éviter l'élimination des déchets dans l'environnement et, si

RC: 97180

Disponible en: https://www.nucleodoconhecimento.com.br/travaux-publics/betonstructurel 
l'on vérifiait la possibilité de son application en remplacement partiel des agrégats naturels, elle éviterait l'extraction d'une partie de ces ressources naturelles, qui se font de plus en plus rares.

Les entreprises productrices de béton ont comme caractéristique l'utilisation des matières premières, c'est-à-dire sans avoir besoin de grands processus de traitement, tels que les sables naturels, qui sont extraits des rivières ou des dépôts, et nécessitent un traitement simple tel que le tamisage pour l'élimination des impuretés et la classification de la taille des particules. Par conséquent, l'utilisation de déchets dans la production de béton a une contribution économique à la fois à l'industrie génératrice de déchets, qui fournit les déchets sans avoir besoin de grands processus de traitement, et aux entreprises productrices de béton, qui bénéficieraient de la haute disponibilité des déchets et de leur faible coût, utilisés comme matière première dans leur production.

L'objectif général de cette étude est d'effectuer la classification préliminaire du résidu de cendres de la chaudière industrielle, de définir son mode d'utilisation possible dans le dosage du béton, d'effectuer des dosages expérimentaux pour vérifier la performance des propriétés de base du béton dosé avec l'utilisation de cendres de chaise, d'évaluer les contributions techniques possibles et, enfin, de conclure à la faisabilité de l'utilisation de ce résidu pour le dosage du béton structurel.

\section{MÉTHODOLOGIE}

La conception initiale de la possibilité de rendre possible l'utilisation de cendres de chaudière pour la production de béton découle de l'idée que, à l'origine, seuls trois matériaux de base étaient utilisés dans la production de béton: le ciment, les agrégats et l'eau. Cependant, pour de meilleures performances à l'état frais et durci, l'utilisation d'additifs chimiques a été initiée. Après un certain temps, l'utilisation de matériaux cimentaires de nature organique a été introduite dans ce contexte en tant

RC: 97180

Disponible en: https://www.nucleodoconhecimento.com.br/travaux-publics/betonstructurel 
qu'ajouts au mélange de béton sous forme de scories granulées, de pozzolans, de fumée de silice et de cendres volantes.

Les raisons initiales d'utiliser ces matériaux étaient généralement économiques : ils coûtaient moins cher que le ciment Portland, souvent parce qu'ils existaient dans des gisements naturels, nécessitant peu ou pas de traitement, d'autres fois parce qu'il s'agissait de sous-produits ou de rejets de procédés industriels (NEVILLE, 1997, p. 81).

« Les cendres volantes sont le résultat de chaudières en train de brûler du charbon dans lesquelles une partie de la matière minérale se regroupe formant des cendres de grille, mais la majeure partie est entraînée par le courant d'échappement du gaz, appelé cendres volantes » (MEHTA; MONTEIRO, 1994).

Cette étude a été réalisée en avril 2021, grâce à la recherche d'innovations pour le segment de la production de béton dans la région d'uberlândia, état de Minas Gerais, où un grand nombre d'industries installées dans cette localité ont été observées, qui utilisaient des chaudières comme moyen de générer de la vapeur dans leurs processus de production et, par conséquent, il y avait une grande disponibilité de cendres de chaudière, ainsi que le grand besoin d'une élimination environnementale adéquate ou de la réutilisation des déchets.

\section{1 ÉCHANTILLON ET CARACTÉRISATION DES CENDRES}

L'échantillon du matériel utilisé dans cette étude a été fourni par une grande industrie installée dans la municipalité d'Uberlândia, dans l'État de Minas Gerais, opérant dans plusieurs secteurs tels que l'agriculture, la nutrition animale, les produits pharmaceutiques et autres. Les cendres résultant du processus de combustion de la biomasse d'eucalyptus sont mélangées à de l'eau pour éviter la dispersion atmosphérique et conduites dans le processus de décantation, où l'échantillon a été prélevé. Par conséquent, le matériau collecté présente une teneur élevée en humidité, vérifiée visuellement par l'eau libre présente superficiellement dans l'échantillon collecté, en plus de l'eau absorbée par le matériau lui-même.

$\mathrm{RC}: 97180$

Disponible en: https://www.nucleodoconhecimento.com.br/travaux-publics/betonstructurel 
Après le prélèvement de l'échantillon et sa conduction au laboratoire des matériaux, le processus de séchage du matériau a été effectué pour commencer sa caractérisation de base. En raison de la teneur élevée en humidité observée dans l'échantillon, il a été nécessaire de commencer le processus de séchage en étalant le matériau extérieur sur une doublure en plastique, évitant ainsi la contamination et les dispersions de celui-ci. Après séchage partiel à l'extérieur, le matériau a été emmené à la serre pour terminer le processus de séchage.

L'indice de finesse a été déterminé par le numéro de tamis 200, selon NBR 11579 ABNT (2012). Au cours de l'exécution de cet essai, il a été observé que dans le matériau retenu dans le tamis numéro 200, la plupart d'entre eux étaient constitués de matériaux fins, mais la présence de particules de plus grandes dimensions de taille de particules a été observée, mais dans une faible proportion. L'indice de finesse trouvé pour le matériau était de $46 \%$. Cet indice a été considéré comme élevé, comparé au ciment Portland, qui a cet indice maximum de $10 \%$, établi par NBR 16697 (ABNT, 2018).

La masse spécifique apparente a été déterminée selon NM 52 ABNT (2009). Les cendres présentaient une masse spécifique apparente de 0,26 g/ $/ \mathrm{cm}^{3}$. Pour Bauer (2008), les agrégats peuvent être classés en fonction de leur poids spécifique. Par conséquent, les cendres ont été classées, à cet égard, comme agrégats légers, et le poids spécifique trouvé était équivalent au poids spécifique des agrégats légers connus, à titre d'exemple d'agrégat léger à la vermicusite, qui a un poids spécifique de $0,3 \mathrm{~g} / \mathrm{cm}^{3}$.

La caractérisation granulométrique a été réalisée selon NBR NM 248 ABNT (2003). Les cendres présentaient une distribution granulométrique selon le tableau 1, module de finesse de 0,74 et dimension maximale de 1,2 $\mathrm{mm}$.

RC: 97180

Disponible en: https://www.nucleodoconhecimento.com.br/travaux-publics/betonstructurel 
Tableau 1 - Distribution granulométrique gris chaudière

\begin{tabular}{|l|l|l|}
\hline $\begin{array}{l}\text { Tamis } \\
\text { (mm) }\end{array}$ & Retenu (\%) & Cumulé (\%) \\
\hline $\mathbf{2 , 4}$ & 0,80 & 0,80 \\
\hline $\mathbf{1 , 2}$ & 2,47 & 3,27 \\
\hline $\mathbf{0 , 6}$ & 5,74 & 9,01 \\
\hline $\mathbf{0 , 3}$ & 12,23 & 21,24 \\
\hline $\mathbf{0 , 1 5}$ & 18,43 & 39,67 \\
\hline Fond & 60,33 & $* * *$ \\
\hline
\end{tabular}

Source : Auteur (2021).

Sur la base des caractéristiques de base vérifiées, nous avons essayé d'attribuer une classification initiale des cendres pour définir leur forme d'application en dosages concrets. Deux possibilités décrites ci-dessous ont été initialement envisagées.

La première possibilité d'utiliser des cendres serait de les incorporer dans le dosage du béton en tant qu'addition, en commençant par référence les ajouts de matériaux comme exemple de silice active, largement utilisée dans la production de béton à haute performance et de potentialiser les propriétés spécifiques du béton, telles que les résistances mécaniques, principalement. Cependant, en raison du profil vérifié des cendres dans la détermination de leur indice de finesse et de leur distribution granulométrique, cette possibilité a été exclue en raison de l'incompatibilité de ses caractéristiques avec ce type d'addition.

Comme deuxième possibilité d'utiliser des cendres, leur introduction possible en remplacement des agrégats dans le dosage du béton a été vérifiée. Les résultats obtenus par rapport au poids spécifique ont été observés et, comme nous l'avons déjà signalé, il a été présenté comme un agrégat léger. Une autre caractéristique

RC: 97180

Disponible en: https://www.nucleodoconhecimento.com.br/travaux-publics/betonstructurel 
analysée, compte tenu de cette possibilité d'utilisation, était la classification des cendres par rapport à la plage granulométrique dans laquelle elles étaient encadrées, sur la base du tableau 2.

Tableau 2 - Classification des sables par aires granulométriques

\begin{tabular}{|l|l|l|l|l|}
\hline $\begin{array}{l}\text { Pourcentages } \\
\text { retenus }\end{array}$ & \multicolumn{2}{|l|}{} \\
\hline Tamis $(\mathbf{m m})$ & $\begin{array}{l}\text { Piste } 1-\text { Très } \\
\text { mince }\end{array}$ & $\begin{array}{l}\text { Piste } 2 \\
\text { Mince }\end{array}$ & $\begin{array}{l}\text { Piste } 3 \\
\text { Moyenne }\end{array}$ & $\begin{array}{l}\text { Piste } \quad 4 \\
\text { Épaisse }\end{array}$ \\
\hline $\mathbf{6 , 3}$ & 0 à 3 & 0 à 7 & 0 à 7 & 0 à 7 \\
\hline $\mathbf{4 , 8}$ & 0 à 5 & 0 à 10 & 0 à 11 & 0 à 12 \\
\hline $\mathbf{2 , 4}$ & 0 à 5 & 0 à 15 & 0 à 25 & 5 à 40 \\
\hline $\mathbf{1 , 2}$ & 0 à 10 & 0 à 25 & 10 à 45 & 30 à 70 \\
\hline $\mathbf{0 , 6}$ & 0 à 20 & 21 à 40 & 41 à 65 & 66 à 85 \\
\hline $\mathbf{0 , 3}$ & 50 à 85 & 60 à 88 & 70 à 92 & 80 à 95 \\
\hline $\mathbf{0 , 1 5}$ & 85 à 100 & 90 à 100 & 90 a100 & 90 à 1 \\
\hline
\end{tabular}

Source : Bauer (2008).

La distribution granulométrique des cendres présentée dans le tableau 1 n'a été entièrement encadrée dans aucune des plages de classification décrites dans le tableau 2. Cependant, des caractéristiques similaires à la distribution classée comme piste 1 - Très mince ont été notées, en s'inscrivant dans cette gamme les pourcentages retenus dans les tamis avec ouverture $2,4 \mathrm{~mm}, 1,2 \mathrm{~mm}$ et $0,6 \mathrm{~mm}$. Dans les autres tamis, les pourcentages conservés sont restés inférieurs à ceux spécifiés dans le tableau 2.Une forte concentration de matériau a été observée au bas de la série de tamis, c'est-à-dire en passant du matériau dans le tamis avec une ouverture de $0,15 \mathrm{~mm}$.

RC: 97180

Disponible en: https://www.nucleodoconhecimento.com.br/travaux-publics/betonstructurel 
Il a donc été vérifié que les cendres présentaient une distribution granulométrique très proche de la bande 1 - Très mince, mais avec un pourcentage significatif de matériau plus mince que prévu dans cette gamme granulométrique, c'est-à-dire tendant vers une plage précédente, s'il y en avait.

Le module de finesse de 0,74 , obtenu par la caractérisation granulométrique des cendres, comme déjà rapporté, a renforcé la classification des cendres dans une plage antérieure à la bande 1 - Très mince, puisque le module de finesse minimale pour la classification dans cette plage serait de 1,35 (BAUER, 2008).

En raison de l'incompatibilité des cendres avec l'indice de finesse des additions traditionnellement utilisées et de leur classification partielle en tant qu'agrégat très mince, nous avons donc choisi d'étudier la possibilité de remplacer des agrégats très minces par des cendres dans le dosage du béton.

\subsection{COURSE STANDARD ET NIVEAUX DE REMPLACEMENT}

La définition du caractère à utiliser comme norme était basée sur les caractéristiques des cendres, préalablement déterminées. Le trait par défaut doit contenir dans sa composition l'agrégat classé comme très mince, qui serait remplacé par du gris.

L'augmentation de la consommation d'eau dans la dose due à l'ajout de cendres a été un point d'attention dans la définition du coup standard, car compte tenu de l'avertissement de Neville (1997) " les cendres de paille de riz ont des formes complexes, selon la plante d'origine et, par conséquent, nécessitent beaucoup d'eau. ", il a été observé le temps nécessaire pour sécher l'échantillon de cendres de chaudière et il a été considéré qu'en raison de son origine et de sa surface spécifique, peut-être que l'ajout de cendres augmenterait la consommation d'eau à la dose par rapport à la dose standard.

Pour lutter contre l'augmentation de la consommation d'eau à des doses avec l'ajout de cendres, II est recommandé par Neville (1997) d'utiliser des additifs

RC: 97180

Disponible en: https://www.nucleodoconhecimento.com.br/travaux-publics/betonstructurel 
superplastifiants pour atteindre la maniabilité attendue. Par conséquent, nous avons choisi de définir le trait standard avec l'utilisation de ce type d'additif.

La course standard a été adoptée avec une résistance à la compression requise de $25 \mathrm{MPa}$ et une diffusion comprise entre 600 et $650 \mathrm{~mm}$, déterminée selon NBR 15823-2 ABNT (2017). Les proportions suivantes ont été trouvées pour le trait massique unitaire: $1: 0,59: 2,21: 1,11: 2,60$, avec un rapport a/c égal à 0,58 , le quantitatif pour le mélange selon le tableau 3 a été obtenu.

Tableau 3 - Course standard 25,0 MPa Diffusion 600 à 650 mm

\begin{tabular}{|l|l|l|}
\hline Ciment & $\mathbf{1 0}$ & $\mathrm{Kg}$ \\
\hline Sable très fin & 5,88 & $\mathrm{Kg}$ \\
\hline Sable moyen & 22,10 & $\mathrm{Kg}$ \\
\hline Gravier 0 & 11,12 & $\mathrm{Kg}$ \\
\hline Gravier 1 & 25,96 & $\mathrm{Kg}$ \\
\hline Additif de plastifiant & 0,035 & $\mathrm{~L}$ \\
\hline $\begin{array}{l}\text { Additif } \\
\text { superplastifiant }\end{array}$ & 0,10 & $\mathrm{~L}$ \\
\hline Eau & 5,8 & $\mathrm{~L}$ \\
\hline
\end{tabular}

Source : Auteur (2021).

L'agrégat nommé " sable très fin » dans le tableau 3 est l'agrégat adopté comme étant remplaçable par les cendres de chaudière. L'agrégat présenté dans sa distribution granulométrique, module de finesse de 1,42, classé dans la gamme granulométrique 1, qui comprend des agrégats avec un module de finesse compris entre 1,35 et 2,25. Par conséquent, il a été classé comme un agrégat très mince (BAUER, 2008).

RC: 97180

Disponible en: https://www.nucleodoconhecimento.com.br/travaux-publics/betonstructurel 
Le remplacement de l'agrégat très fin par des cendres a été partiellement effectué, c'est-à-dire en adoptant des niveaux de substitution aléatoires et progressifs, afin de provoquer l'observation des influences possibles causées par le remplacement de l'agrégat par des cendres de chaudière. À titre de départ, des niveaux de substitution de $10 \%, 20 \%$ et $30 \%$ ont été adoptés sur la masse agrégée très fine présente dans la posologie du caractère standard déjà présentée dans le tableau 3.

Le tableau 4 montre les caractéristiques unitaires de masse adoptées pour chaque teneur de substitution établie.

Tableau 4 - Traces unitaires massiques retenues en fonction de la teneur en remplacement de l'agrégat très fin par cendres de chaudière

\begin{tabular}{|l|l|}
\hline $\begin{array}{l}\text { Contenu de remplacement agrégé pour } \\
\text { les cendres }\end{array}$ & $\begin{array}{l}\text { Caractéristique de l'unité de } \\
\text { masse }\end{array}$ \\
\hline $\mathbf{1 0} \%$ & $1: 0,06: 0,53: 2,21: 1,11: 2,60 \mathrm{a} / \mathrm{c}$ \\
& $=0,58$ \\
\hline $\mathbf{2 0} \%$ & $1: 0,12: 0,47: 2,21: 1,11: 2,60 \mathrm{a} / \mathrm{c}$ \\
& $=0,58$ \\
\hline $\mathbf{3 0} \%$ & $1: 0,18: 0,41: 2,21: 1,11: 2,60 \mathrm{a} / \mathrm{c}$ \\
& $=0,58$ \\
\hline
\end{tabular}

Source : Auteur (2021).

\subsection{DOSAGES EXPÉRIMENTAUX ET ÉVALUATIONS EFFECTUÉES}

Des dosages expérimentaux ont été effectués en laboratoire à l'aide d'une bétonnière électrique stationnaire. Tout d'abord, le caractère standard a été dosé dans le tableau 3 , puis les autres caractères avec les niveaux de substitution respectifs adoptés, présentés dans le tableau 4.

RC: 97180

Disponible en: https://www.nucleodoconhecimento.com.br/travaux-publics/betonstructurel 
Comme principal critère d'évaluation, les influences possibles de l'introduction de cendres de chaudière dans les dosages par rapport aux propriétés ont été adoptées: maniabilité du béton à l'état frais, densité et résistance à la compression.

Pour évaluer l'influence de l'introduction de cendres sur le dosage par rapport à la maniabilité du béton à l'état frais, le dosage a été effectué à tous les dosages pour déterminer la consistance initiale par la réduction du tronc du cône, selon NBR 16889 ABNT (2020). La consistance initiale adoptée en standard se situe entre 40 $\mathrm{mm}$ et $60 \mathrm{~mm}$. Après avoir atteint la consistance initiale, l'additif superplastifiant a été ajouté aux dosages dans le but d'augmenter la maniabilité et d'atteindre la propagation entre 600 et $650 \mathrm{~mm}$, qui a été déterminée dans tous les dosages selon NBR 15823-2 ABNT (2017).

En ce qui concerne la densité, elle a été déterminée selon NBR 9833 ABNT (2008).

L'évaluation de la résistance à la compression a été réalisée par moulage d'éprouvettes cylindriques de dimensions 10x20mm, selon NBR 5738 ABNT .;(2016). Deux échantillons de chaque série ont été soumis à l'essai pour déterminer la résistance axiale à la compression à l'âge de 7 jours, 14 jours et 28 jours, selon NBR 5739 ABNT (2018).

\subsection{RÉSULTATS ET DISCUSSIONS}

Dans le dosage du caractère standard, il a été observé que l'eau prédite dans le caractère était suffisante pour atteindre la consistance initiale, car l'indice de consistance initial de $52 \mathrm{~mm}$ a été obtenu. Ainsi, l'additif superplastifiant a été introduit dans le mélange et le temps de mélange de 8 minutes après le dosage de l'additif superplastifiant a été établi. Ce temps de mélange est le temps moyen déterminé par le fabricant pour envisager le plein effet de l'additif. Après le temps de mélange, l'étalement a été déterminé et $620 \mathrm{~mm}$ ont été obtenus en conséquence, c'est-à-dire dans les paramètres précédemment établis.

RC: 97180

Disponible en: https://www.nucleodoconhecimento.com.br/travaux-publics/betonstructurel 
Après le dosage de trace standard, la trace a été dosée avec une teneur de remplacement de $10 \%$ de l'agrégat très mince par des cendres de chaudière. Dans ce dosage, en utilisant la même quantité d'eau par rapport au trait standard, un indice de consistance initial de $49 \mathrm{~mm}$ a été obtenu. II a donc été noté que l'introduction de cendres a provoqué une légère baisse de la consistance initiale du béton. Cependant, étant toujours dans les paramètres initialement établis, l'additif superplastifiant a été dosé et en respectant le temps standard de 8 minutes pour le mélange, l'étalement a ensuite été déterminé et $600 \mathrm{~mm}$ d'ouverture ont été obtenus.

Dans l'ordre, la trace a été dosée avec une teneur de remplacement de $20 \%$ de l'agrégat très mince par des cendres de chaudière. Avec la même quantité d'eau utilisée dans les dosages précédents, l'indice de consistance initial de $50 \mathrm{~mm}$ a été obtenu. Avec le dosage de l'additif superplastifiant et la fin du temps de mélange standard, l'ouverture de $600 \mathrm{~mm}$ a été étalée, c'est-à-dire un comportement très similaire au dosage précédent, avec une teneur de remplacement de $10 \%$ de l'agrégat très fin par les cendres de chaudière.

Enfin, la trace a été dosée avec une teneur de remplacement de 30\% de l'agrégat très fin par des cendres de chaudière. En maintenant la quantité d'eau des doses précédentes, l'indice de consistance initial de $38 \mathrm{~mm}$ a été obtenu, c'est-à-dire en dessous du paramètre initial établi. Dans ce cas, il a été observé que l'introduction de cendres dans le mélange interférait dans la consistance initiale comme l'avait averti Neville (1997), mais nous avons opté pour la séquence posologique car l'indice de consistance initial obtenu était très proche de la limite inférieure établie, qui était de $40 \mathrm{~mm}$. Après le dosage de l'additif superplastifiant et la fin du temps de mélange, $560 \mathrm{~mm}$ ont été étalés. Par conséquent, en dessous du paramètre initialement.

Dans le cas spécifique du dosage avec une teneur de remplacement de $30 \%$ de l'agrégat très fin par les cendres de chaudière, il a été décidé d'augmenter le dosage de l'additif superplastifiant afin d'obtenir le miroir minimum prédéterminé. Par

RC: 97180

Disponible en: https://www.nucleodoconhecimento.com.br/travaux-publics/betonstructurel 
conséquent, la posologie de l'additif superplastifiant a été augmentée de $1 \%$ à 1,5\% sur le poids du ciment. Après cet ajout d'additif au mélange et respecté à nouveau le temps de mélange pour l'action totale de l'additif, l'étalement de $610 \mathrm{~mm}$ a été obtenu, c'est-à-dire en s'adaptant aux paramètres préétablis.La maniabilité est une propriété très importante, car " un mélange de béton qui ne peut pas être facilement jeté ou dense dans son intégralité ne fournira pas les caractéristiques de résistance et de durabilité attendues » (MEHTA; MONTEIRO, 1994).

Les densités trouvées selon NBR 9833 ABNT (2018) sont présentées dans le tableau 5.

Tableau 5 - Densités déterminées

\begin{tabular}{|l|l|}
\hline $\begin{array}{l}\text { Identification de la } \\
\text { série }\end{array}$ & Densité $\left(\mathbf{k g} / \mathrm{m}^{\mathbf{3}}\right)$ \\
\hline Course standard & 2335 \\
\hline Tiret avec $\mathbf{1 0} \%$ de gris & 2078 \\
\hline Tiret avec $\mathbf{2 0} \%$ de gris & 2070 \\
\hline Tiret avec $\mathbf{3 0} \%$ de gris & 2066 \\
\hline
\end{tabular}

Source : Auteur (2021).

Il a été observé qu'il y avait une baisse de densité de $11 \%$, comparant la trace standard à la trace avec un contenu de remplacement de $10 \%$ de l'agrégat très fin par les cendres de chaudière. Pour les caractères suivants, avec des niveaux de substitution de $20 \%$ et $30 \%$, les diminutions de densité enregistrées étaient de l'ordre de 0,4\% et 0,2\%, respectivement, en comparant le caractère analysé au trait qui l'a précédé, compte tenu de l'ordre progressif et progressif du contenu du remplacement de l'agrégat très mince par des cendres de chaudière.

Les résultats obtenus lors des essais visant à déterminer la résistance axiale à la compression sont présentés dans le tableau 6.

$\mathrm{RC}: 97180$

Disponible en: https://www.nucleodoconhecimento.com.br/travaux-publics/betonstructurel 
Tableau 6 - Résistance à la compression (MPa) par série

\begin{tabular}{|l|l|l|l|}
\hline $\begin{array}{l}\text { Résistance série/âge/compression } \\
\text { (MPa) }\end{array}$ & $\mathbf{7}$ jours & $\mathbf{1 4}$ jours & $\mathbf{2 8}$ jours \\
\hline Course standard & 18,1 & 21,0 & 30,3 \\
\hline Tiret avec 10\% de gris & 14,6 & 18,0 & 19,6 \\
\hline Tiret avec 20\% de gris & 15,2 & 18,3 & 20,2 \\
\hline Tiret avec 30\% de gris & 14,6 & 18,5 & 19,8 \\
\hline
\end{tabular}

Source : Auteur (2021).

II a été vérifié par les résultats obtenus que les dosages avec utilisation de cendres de chaudière présentaient des résistances à la compression inférieures au mélange standard adopté dans tous les âges testés. La baisse moyenne de résistance à la compression des mélanges utilisant des cendres de chaudière par rapport au mélange standard a été présentée comme indiqué dans le tableau 7.

Tableau 7 - Pourcentage de chute de la résistance à la compression par rapport à la course standard

\begin{tabular}{|lll|l|l|l|}
\hline Trait/Âge & $\mathbf{7}$ jours & $\mathbf{1 4}$ jours & $\mathbf{2 8}$ jours \\
\hline $\begin{array}{l}\text { Tiret avec } \\
\text { gris }\end{array}$ & $\mathbf{1 0} \%$ de & $19 \%$ & $14 \%$ & $35 \%$ \\
\hline $\begin{array}{l}\text { Tiret avec } \\
\text { gris }\end{array}$ & $\mathbf{2 0} \%$ de & $16 \%$ & $13 \%$ & $33 \%$ \\
\hline $\begin{array}{l}\text { Tiret avec } \\
\text { gris }\end{array}$ & & & & \\
\hline
\end{tabular}

Source : Auteur (2021).

$\mathrm{RC}: 97180$

Disponible en: https://www.nucleodoconhecimento.com.br/travaux-publics/betonstructurel 
Il a été observé que dans tous les traits avec l'utilisation de cendres, le pourcentage de baisse de la résistance à la compression par rapport à la course standard se comportait de manière similaire indépendamment de la teneur de substitution utilisée.

En ce qui concerne la couleur des bétons dosés, il a été constaté que, tant à l'état frais que durci, la teneur en agrégats très fins remplacés par des cendres de chaudière influençait évidemment cet aspect visuel, car en raison de la couleur des cendres de chaudière similaire à celle du ciment Portland, les bétons avec des niveaux plus élevés d'utilisation de cendres ont été visuellement présentés comme du béton avec une consommation de ciment plus élevée, c'est-à-dire qu'avec une couleur plus foncée, plus le contenu de remplacement adopté est élevé.

\section{CONSIDÉRATIONS FINALES}

Les cendres de chaudière industrielle ont été classées comme légères et avec une plage granulométrique légèrement inférieure à la plage très mince, les deux classifications sont appliquées aux agrégats, de sorte que son application a été conclue comme agrégat léger et très mince.

Avec une augmentation de la teneur en agrégats remplacés par des cendres de chaudière industrielle dans le dosage, il y a eu une influence sur la maniabilité du béton, observée par la réduction de la réduction initiale et sur la diffusion finale. Par conséquent, il a été conclu qu'il importait d'adopter la méthode de remplacement partiel de l'agrégat pour l'utilisation de cendres de chaudière industrielle, puisque le remplacement total de l'agrégat a été adopté, il y aurait peut-être un impact encore plus grand sur cette propriété du béton à l'état frais.

L'importante contribution de l'additif superplastifiant dans les dosages avec l'utilisation de cendres de chaudière industrielles a été conclue, dans le but de compenser la perte de maniabilité causée par l'utilisation de cendres de chaudière. II

RC: 97180

Disponible en: https://www.nucleodoconhecimento.com.br/travaux-publics/betonstructurel 
a été entendu qu'avec des additifs de plastifiants, il ne serait pas possible d'obtenir une maniabilité satisfaisante dans les bétons dosés avec les cendres de chaudière industrielle en raison de la nature des cendres et de leur grande surface spécifique, ce qui entraîne une consommation d'eau plus élevée dans les dosages.

En ce qui concerne la densité, il a été conclu qu'avec la substitution de l'agrégat par des cendres de chaudière industrielle, il y avait une diminution de la densité liée à l'augmentation de la teneur de remplacement, c'est-à-dire que plus la teneur en remplacement de l'agrégat par les cendres de chaudière industrielle était élevée, plus la densité vérifiée était faible. II a été jugé très important dans les dosages futurs d'observer l'influence causée par l'utilisation de cendres de chaudière industrielle dans les dosages par rapport à cette propriété concrète.

La performance de la résistance à la compression des bétons dosés avec des cendres de chaudière industrielle était similaire, quelles que soient les variations du contenu de remplacement de l'agrégat. Cependant, il y a eu une diminution de cette propriété par rapport au caractère standard, c'est-à-dire sans l'utilisation du résidu. Par conséquent, cette réduction de la résistance doit être envisagée dans les dosages futurs lorsqu'il s'agit de doser à dose par rapport sans l'utilisation de résidus.

Il a donc été conclu que l'utilisation de cendres de chaudière industrielle dans la mesure du béton structurel est réalisable, car des caractéristiques satisfaisantes ont été obtenues en ce qui concerne la maniabilité et la performance de la résistance à la compression selon des paramètres normatifs. Par conséquent, grâce à cette étude, il a été possible d'identifier les comportements initiaux du béton dosé avec des cendres de chaudière et de prouver la faisabilité de l'utilisation de ce résidu.

Il est entendu qu'il sera nécessaire de poursuivre la recherche d'améliorations, en assurant la bonne performance du béton à l'état frais et durci, ainsi que pendant la durée de vie utile des bâtiments.

$\mathrm{RC}: 97180$

Disponible en: https://www.nucleodoconhecimento.com.br/travaux-publics/betonstructurel 


\section{RÉFÉRENCES}

Associação brasileira de normas técnicas. NBR 11579: Cimento Portland Determinação do índice de finura por meio da peneira 75 um ( $n^{\circ}$ 200). Rio de Janeiro, 2012.

Associação brasileira de normas técnicas. NBR 15823-2: Concreto autoadensável: Parte 2 - Determinação do espalhamento, do tempo de escoamento e do índice de estabilidade visual - Método do cone de Abrams. Rio de Janeiro, 2017.

Associação brasileira de normas técnicas. NBR 16889: Concreto - determinação da consistência pelo abatimento do tronco de cone. Rio de Janeiro, 2020.

Associação brasileira de normas técnicas. NBR 5738: Concreto - procedimento para moldagem e cura de corpos de prova. Rio de Janeiro, 2016.

Associação brasileira de normas técnicas. NBR 5739: Concreto - ensaio de compressão em corpos de prova cilíndricos. Rio de Janeiro, 2018.

Associação brasileira de normas técnicas. NBR NM 248: Agregados - Determinação da composição granulométrica. Rio de Janeiro, 2003.

Associação brasileira de normas técnicas. NM 52: Agregados - Determinação da massa unitária e volume de vazios. Rio de Janeiro, 2009.

Associação brasileira de normas técnicas. NBR 9833: Concreto fresco Determinação da massa específica, do rendimento e do teor de ar pelo método gravimétrico. Rio de Janeiro, 2008.

Bauer, Luiz Alfredo Falcao. Materiais de construção. Rio de Janeiro: LTC, 2008.

$\mathrm{RC}: 97180$

Disponible en: https://www.nucleodoconhecimento.com.br/travaux-publics/betonstructurel 
Cavalcante, Zedequias Vieira. A Importância da Revolução Industrial no Mundo da tecnologia. Unicesumar. Maringá, 2011. Encontro Internacional de Produção Científica. Disponível em: https://www.unicesumar.edu.br/epcc-2011/wpcontent/uploads/sites/86/2016/07/zedequias_vieira_cavalcante2.pdf. Acesso em: 10 abr. 2021.

Ganzala, Gabryelly Godois. A Industrialização, impactos ambientais e a necessidade de desenvolvimento de políticas ambientais sustentáveis no século XXI. 2018. Centro Universitário Internacional - UNINTER. Disponível em: https://repositorio.uninter.com/bitstream/handle/1/295/1355104\%20\%20GABRYELLY\%20GODOIS\%20GANZALA.pdf?sequence=1 \&isAllowed=y. Acesso em: 4 abr. 2021.

MEHTA, P. K. e MONTEIRO, P. J. M. Concreto: Estrutura, Propriedades e Materiais. São Paulo: Editora Pini, 1994.

Neville, Adam Matthew. Propriedades do concreto. São Paulo: Editora Pini, 1997.

Soumis: Août, 2021.

Août : Septembre 2021.

RC: 97180

Disponible en: https://www.nucleodoconhecimento.com.br/travaux-publics/betonstructurel 